\title{
Challenges for cerebrovascular disease
}

\author{
Werner Hacke ${ }^{1 *}$ and Lou Caplan ${ }^{2 * *}$ \\ 'Department of Neurology, University of Heidelberg, Heidelberg, Germany \\ ${ }^{2}$ Beth Israel Deaconess Medical Center, Boston, MA, USA \\ Correspondence: *werner.hacke@med.uni-heidelberg.de; **Icaplan@bidmc.harvard.edu
}

\section{ACUTE STROKE CARE: HOW CAN MODERN STROKE CARE BE MADE AVAILABLE TO MORE PATIENTS?}

Much progress has been made in acute stroke management during the last 20 years. Today we can offer organized stroke unit care in many regions in Europe and some in the United States and Canada; we have an approved acute medical treatment for ischemic stroke, i.v. rtPA. Clot retrieval devices are approved and available and we have evidence-based treatment strategies for critically ill stroke patients. A number of promising new approaches, still await formal evidence, although they are already used quite frequently.

However, this progress is not available everywhere. There are large disparities between what is known and what can be offered in many countries and regions throughout the world. The standard of care in some regions is far from routine in others, or may be available only at selected places for very few privileged people. Unfortunately, there is no uniform way in which the application and expansion of what we know in acute stroke management can be promoted throughout the world. Rather, individual routes must be defined with respect to availability of resources, standards of training and teaching, and priorities in health economics.

The establishment of Stroke Units and Stroke Centers has been the most valuable contribution to the field of acute stroke management. It is not only the single most effective intervention for the vast majority of stroke patients, but it is also a major factor towards increasing the number of patients receiving thrombolytic therapy. Together with pre-hospital system organisation and access to rehabilitation and secondary prevention, these are the most important columns on which the expansion of stroke treatment is built.

Once "Stroke" has reached political awareness, implementation of care sys- tems proceeds more smoothly. The political will to improve stroke care will allow new activities in the field of stroke to be launched. Nation- or statewide documentation, standards, and quality control instruments can be implemented, and more financial resources will be made available for the development of stroke management and prevention. Stroke care is expensive, and stroke care has to be paid out of the shrinking budgets of general health care. Increasing the resources for one area means cutting resources in others, not an action that will be warmly welcomed by colleagues in other fields.

Although capitalizing on existing therapies is well justified, flexibility has to be built into the system to facilitate the successful investigation and application of new diagnostic and therapeutic approaches as they are developed. By doing so, the stroke care system will remain flexible and will be amenable to incorporate advances that will continue to improve the care of the stroke patient.

We propose a stepwise approach to improvement of stroke care.

\section{STEP 1: INCREASE STROKE AWARENESS}

Main barriers for stroke treatment are the lack of the recognition of stroke signs and the lack of a sense of urgency to seek help. Campaigns of stroke awareness can decrease the time from symptoms onset to the hospital and increase the number of treated patients. Multi modal media campaigns that improve public information about acute stroke signs and the urgency to call pre-hospital emergency medical systems must be developed and implemented in the different regions. Public education campaigns should encourage not only affected individuals, but also family and bystanders to call the pre-hospital emergency medical system if they observe an individual having signs of a possible stroke.

\section{STEP 2: IMPROVE EMERGENCY STROKE CARE}

Activating the prehospital emergency medical system in acute stroke care is associated with a shorter delay to arrival at hospital. The training of ambulance team and dispatchers in prehospital recognition of stroke as an emergency and the recognition of stroke signs increases the number of patients arriving earlier at hospital. Training of pre-hospital emergency medical systems team to recognize stroke and to bring acute stroke patients directly to designated stroke centers is key to improvement of overall stroke care. Regional networks of stroke care between pre-hospital emergency medical systems and stroke centers must be established.The same telephone number in whole country to activate the pre-hospital emergency medical system is needed (the European 112 and USA 911campaigns).

\section{STEP 3: ESTABLISHMENT OF STROKE CENTERS AND STROKE UNITS TO ASSIST ACUTE STROKE PATIENTS AS A PRIORITY}

Efforts should be made to establish Stroke Center hospitals with organized Stroke Units in locations nearby and accessible to all stroke patients to reduce the burden of stroke in the world. These specialized centers should be organized according to local and regional needs and classified in different levels of complexity according to the resources and treatments available in each. Stroke Centers should implement evidence based protocols, including thrombolytic therapy. Hospitals in each city or region should be identified as stroke centers with organized Emergency Department and Stroke Unit care in accordance with Local Health Authorities. Stroke Units and Stroke Centers must be classified and certified according to standard definitions. Quality standards and minimum quantities of interventions and procedures must be established. Specialized staff must be trained with standardized protocols includ- 
ing the implementation of thrombolytic therapy for acute ischemic stroke. Quality control instruments must be implemented and made compulsory.

We challenge authors to submit reports about translation of knowledge about stroke and its treatments into practical delivery of stroke care to patients.

\section{IMPROVING THE APPLICABILITY OF EVIDENCE-BASES TO THE CARE OF INDIVIDUAL PATIENTS AND INCLUDING FLEXIBILITY AND CONTEXT INTO GUIDELINES}

Everyone desires their approach to diagnosis and treatment to be evidence-based. Although some define evidence-based as determined only by the results of prospective randomized double-blind therapeutic trials, most physicians use different types of evidence, not all equal, to decide on treatment for individual patients. Randomized trials have definite limitations. The major theoretical limitation of trials is the issue of numbers vs specificity. For trials to yield statistically valid and important results, they must include a very large quantity of patients - numbers. For the results to be useful to practicing physicians, the data must be specifically applicable to individual patients who have the condition studied. To include enough patients, the condition studied must be common and usually multiple physicians at many medical centers must be used. A single doctor or center would have too few patients or would take an unacceptably long time to enroll and study the number of required patients. To achieve numbers, a "lumping" strategy must predominate over "splitting."

Information from trials must be weighted according to the context of specific treatment decisions. Conducting trials is different from caring for individual patients. In trials, the same treatments are given to all eligible patients depending only on randomization. Departure from the specified treatment makes the results difficult to interpret. In the clinic, doctors treat individual patients.

Courts continuously wrestle with the issue of the applicability of general laws and precedence from prior individual cases to the case being considered. For example, although there may be general evidence that aspirin has some effectiveness against brain ischemia, there may be no evidence that it is effective or ineffective for a given vascular disorder unless it has been specifically tested for that disorder. Even when it has been tested for that disorder, coexisting factors in a particular patient often complicate the decision about whether or not to use a particular agent in a particular patient at a particular time. Therapeutic decisions are often complex and require experienced physicians. Treatment decisions for individual patients cannot be made by protocols, rules, or computer searches. Evidence from trials, past experience, and intimate detailed knowledge of the patient, the diseases, and the wishes and desires of all concerned are required to make difficult therapeutic decisions. Non-physicians (commonly insurers and payers) should not be allowed to control this decision making process by mandating guidelines that are influenced mostly by cost considerations.

The challenge is two-fold.

1) How to make trials and trial results more useful for treatment of individual patients?

One key strategy to make results more useful is defining meaningful subgroups within a trial. Using cerebrovascular disease as an example, this would require mandating that all patients have thorough analyses of potential cardio- cervico-cranial vascular, and hematological causes of brain ischemia, and reporting of the nature, location and severity of steno-occlusive lesions and brain infarcts and hemorrhages. Subgroups can be managed either by prospective stratification, that is, by randomizing patients using predetermined criteria (e.g., sex, race, age, vascular lesions, stroke subtypes) to ensure that subgroups will be relatively equally represented in the different treatment groups, or by analyzing the treatment results by subgroup determinants that have been prospectively defined but the subgroups must also be very large to satisfy statisticians. An issue that has been problematic is paying for such large trials. Mandating a thorough evaluation means that the funding agency mmust pay fort hat evaluation-greatly increasing the cost of evaluation of each patient by the funding agency. In fact, many centers now evaluate patients thoroughly as part of the normal work-up.

An alternative is to have trials that study only subgroups, for example trials that consider only patients with atrial fibrillation or stenosis of the basilar artery, or ischemia due to penetrating artery disease ("lacunar stroke").

2) How to make guidelines more flexible and more related to the context of the individual patient being treated?

Some guidelines slavishly repeat the inclusions and exclusions of trials and give absolute rules applicable to all patients. Payers use these absolutes to deny payment, and lawyers use these guidelines in medical malpractice suits against physicians who deviate from the absolutes. Instead, guidelines should begin by stating the theory behind selection of treatment and variables to be considered. Guidelines should disclose the populations studied in trials that were used to generate the recomendations, and should acknowledge which if any subgroups were specifically studied. What if language is helpful in giving context to decisions; for example in thrombolytic guidelines, What if a patient is seen after a defined time (such as $4.5 \mathrm{~h}$ ) and has an intracranial occlusive lesion and a significant diffusion/perfusion mismatch. Physician judgement and patient and family input will always be applicable for individual patient decisions and language acknowledging that reality should be included in guidelines.

We challenge authors to submit articles about the conduct of trials, definition and management of stroke subgroups and about stroke guidelines.

\section{LEARNING MORE ABOUT RECOVERY FROM STROKE AND THE EFFECTIVENESS OF VARIOUS REHABILITATION STRATEGIES}

Stroke will never be completely conquered by hyperacute treatment after symptoms of neurological dysfunction develop. A great many stroke patients will never arrive at major stroke centers in time for hyperacute treatment. Even then many of those treated will have residual neurological deficits. Little of scientific valid- 
ity is now known about recovery from neurological deficits including stroke. The development of improved diagnostic technology including functional MRI and newer treatment modalities including magnetic and electrical stimulation provide an opportunity to study more scientifically how individuals recover and what strategies facilitate or impede natural recovery.
We challenge authors to submit material about stroke recovery and its treatment.

\section{CLINICAL AND EPIDEMIOLOGICAL \\ FEATURES OF STROKE AND} CEREBROVASCULAR DISEASE AND THEIR DIAGNOSIS AND MANAGEMENT Frontiers in Stroke is interested in receiving new information on all aspects of cerebrovascular disease.
Received: 16 February 2010; accepted: 18 February 2010; published online: 31 March 2010.

Citation: Hacke W and Caplan L (2010) Challenges for cerebrovascular disease. Front. Neur. 1:3. doi: 10.3389/ fneur.2010.00003

This article was submitted to Frontiers in Stroke, a specialty of Frontiers in Neurology.

Copyright $(0) 2010$ Hacke and Caplan. This is an open-access article subject to an exclusive license agreement between the authors and the Frontiers Research Foundation, which permits unrestricted use, distribution, and reproduction in anymedium, provided the original authors and source are credited. 